\title{
Los profesionales de secundaria, como factores de riesgo en el síndrome de Burnout
}

\section{Secondary School Professionals: Burnout Syndrome's Risk Factors}

\author{
Giselle León León ${ }^{1}$ \\ División de Educología \\ Centro de Investigación y Docencia en Educación \\ Universidad Nacional \\ Heredia, Costa Rica \\ leongiselle@hotmail.com
}

Recibido 21 de julio de $2010 \bullet$ Aceptado 31 de agosto de 2010

\begin{abstract}
Resumen. Este artículo aborda el síndrome de Bournout, entendido como un trastorno de la adaptación ante el estrés crónico laboral que logra desencadenar síntomas físicos y psicológicos, los cuales dañan significativamente la ejecución profesional de los individuos que prestan servicios, en este particular, el trabajo de los docentes de secundaria, los cuales se describen como vulnerables por atender a adolescentes, quienes requieren mayor comunicación, atención y guía por las característica propias de esa etapa. Además se describen algunos factores que pueden incidir en el docente, desde una perspectiva individual como por ejemplo estrés, rol, edad, estado civil, entre otros. Asímismo factores laborales tales como baja implicación, sobre carga, burocracia, ambiente, consecuencias sobre el individuo y sobre la institución y medidas para prevenirlo.
\end{abstract}

Palabras claves. Estrés laboral, docentes secundaria, factores de riesgo, prevención.

Abstract. This paper discusses the Burnout syndrome, understood as an adaptation disorder due to chronic occupational stress, triggering both physical and psychological symptoms that significantly affect the professional performance of individuals who work in services. Secondary school teachers consider themselves as vulnerable to this syndrome, since they work with teenagers that demand better communication, attention and guidance due to the normal characteristics of the age. It also describes some of the factors that may affect teachers, either from an individual perspective (stress, role, age and civil status, among others); or from a labor perspective (low involvement, work overload, bureaucracy, environment, individual and institutional consequences and preventive measures).

Keywords. Occupational stress, high school teachers, risk factors, prevention.

\footnotetext{
Máster en Administración Educativa (2006), bachiller en la Enseñanza de las Ciencias en la Universidad Nacional (2002) y Licenciatura en Ciencias de la Educación con Énfasis en Didáctica de Ciencias (2003). Actualmente académica de la división de Educología y coordinadora por parte de la UNA del proyecto Mejora de la Oferta Educativa en Gestión Ambiental. Dentro de las últimas publicaciones: Educación para el cambio: protección del planeta, El síndrome de Burnout, una realidad en educación, La gestión ambiental como estrategia educacional (experiencia del proyecto: Mejora de la oferta educativa en gestión ambiental-Sarapiquí).
} 


\section{Introducción}

En nuestra sociedad, la estructura educativa tiene como función la socialización y transformación de un individuo en el seno de una cultura. Gran parte de esta responsabilidad la tienen las instituciones educativas, que por espacio de varios años lo están formando en diferentes áreas formales de la educación o lo capacitan para un mercado laboral. Es así como la mayor parte de la transformación de la cultura depende de los actores dentro de las instituciones educativas, quienes mediante diferentes procesos y mecanismos de enseñanza y aprendizaje reconstruyen lo esencia de los conocimientos, las ideas, las creencias, las normas, los valores, los hábitos y hasta los sentimientos y acciones, en suma, la educación se constituye en un promotor de valores y un regulador del desarrollo de las personas que conforman la sociedad.

De este modo, uno de los actores en este proceso son los profesionales de Educación Media, que con sus acciones y conductas construyen el conocimiento, permitiendo generar una identidad cultural que se renueva continuamente, se transforma en ciertos espacios, pero también puede estancarse, de aquí la importancia de tener docentes sanos física y mentalmente, ya que la transmisión de gran parte de la cultura se da por medio de la comunicación directa entre el profesor y los estudiantes.

Los jóvenes que acuden a los colegios de secundaria son adolescentes que aún están construyendo su identidad, para poder hacer frente, en un futuro, a una realidad social más compleja que les exigirá conocimientos especializados para lograr un rol de trabajador y si no cuenta con las competencias básicas en los espacios de formación que la sociedad ha estructurado, entonces correrá el riesgo de no realizarse de forma integral, autónoma y equilibrada.

Por otra parte, vivimos en una sociedad que impone un ritmo de trabajo acelerado, que muchas veces impide realizar plenamente lo que se espera de los individuos y que, al mismo tiempo, puede repercutir en la salud y en la calidad de vida de los trabajadores. Una de las consecuencias de esto es el estrés que, cuando se presenta de manera continua y prolongada, origina un problema de salud laboral, llamado síndrome de Burnout o de quemado. Este síndrome ha sido estudiado desde el primer tercio del Siglo XX, en el terreno de las instituciones del sector salud y, en la última década, también en el sector educativo. Vale la pena señalar que en Costa Rica no existen muchas publicaciones al respecto.

Este síndrome se entiende como un trastorno de la adaptación ante el estrés crónico laboral que logra desencadenar síntomas físicos y psicológicos y daña significativamente la ejecución profesional de los individuos que prestan servicios en aquellas áreas donde una de las principales labores es cuidar intereses y satisfacer necesidades de otras personas.

Se caracteriza por presentar síntomas de agotamiento emocional, identificadas con el sentimiento de estar excedido en los recursos personales, es decir, sentirse abrumado emocionalmente; la despersonalización comprende la respuesta impersonal y actitudes de irritabilidad; por último, la sensación de disminución de logro personal, con el sentimiento de pérdida de competencia y de éxitos en el trabajo. Se acompaña de síntomas físicos y conductuales individuales, de problemas en relaciones interpersonales e incluso del abuso de sustancias. Además, se relaciona con situaciones laborales y organizacionales, yales como la presencia de tensión en el trabajo e insatisfacción laboral (Tonon, 2003).

Por lo descrito anteriormente, a continuación se presenta una descripción de este síndrome, para que los docentes, en especial los de Enseñanza Media, puedan conocer y prevenir el síndrome de Burnout o estar quemado. 


\section{Los factores de riesgo en los docentes}

El docente que manifiesta el síndrome de Burnout es, frecuentemente, impredecible en su conducta y las contradicciones son casi siempre la norma de su actividad. En este sentido, a la vez que siente la necesidad imperiosa de culpar a alguien por lo que pasa, también precisa olvidar al máximo todo lo relacionado con su trabajo, estudiantes, planes, documentos pendientes, entre otros.

Para los profesores que llegan a este estado, los conflictos entre la familia y su profesión, los dos sistemas en los que interactúan, son especialmente constantes e intensos. Está muy extendido el estado emocional caracterizado por el miedo, la hipervigilancia y la preocupación permanente. En muchos casos, los sentimientos, ansiedad y agotamiento, se confunden o se alternan provocando, a su vez, los citados problemas de relaciones interpersonales.

El profesor y la profesora en estado de Burnout, agobiado por las demandas y agotado por su trabajo, muestra un carácter irritable que puede resultar insoportable para las otras personas, tanto en el entorno laboral como cuando la jornada laboral ha terminado. Poco a poco, la persona se va sintiendo afectada, y va cambiando sus actitudes hacia el trabajo y hacia los compañeros y compañeras con los que trabaja, hasta que el proceso se evidencia cada día más. De una manera lenta, pero progresiva, estos profesionales pueden abandonar sus relaciones habituales, dedican menos atención a su familia, muestran una insensibilidad notable, cierran los canales de comunicación con otras personas y se desentienden de los demás.

En general, los profesores y las profesoras con Burnout perciben la enseñanza como un trabajo muy exigente y que no recompensa suficientemente, por lo que los acontecimientos son interpretados de manera negativa y pesimista, percepción que es trasmitida a los educandos.

Actualmente, los docentes se encuentran en una situación compleja y delicada. Basta poner atención a los medios de comunicación (televisión, prensa, Internet, etc.) para observar como se les culpabiliza de los problemas, principalmente de los que ocurren dentro del contexto escolar, por ejemplo, las relaciones docente-educando (relaciones personales, falta de asimilación de los contenidos curriculares ente los estudiantes, entre otros), así como los referentes a cuestiones de tipo social, (violencia, desprestigio, drogas, situaciones familiares, entre otros).

En este sentido, se puede destacar la situación del contexto socio-escolar, el cual deriva fácilmente en frustración y desmotivación ante la evidente falta de estrategias disponibles para reorientar los conflictos y la convivencia docente. Iniciar el trabajo cada nuevo día, especialmente los lunes, se visualiza como una carga difícilmente soportable, la que, con frecuencia, conduce al abandono de la profesión o, en su defecto, a permanecer incapitados por factores emocionales, dolores de cabeza, tención u otros padecimientos anexados al síndrome.

Las tendencias actuales, en el ámbito de la educación, orientan hacia las interacciones humanas de calidad, la creación de climas y ambientes más propicios y adecuados, para que tales interacciones sean positivas, satisfactorias y puedan surtir los efectos deseados. El docente establece una relación intencionada desde su perspectiva de adulto con el individuo que ha de desarrollar armónicamente. En todo este proceso, el docente tiene la misión de guía, orientador, facilitador y evaluador: debe garantizar que los educandos tomen parte activa y responsable en el mismo. Esto representa en sí mismo, una complicada dificultad, puesto que el proceso sólo tendrá éxito si los dos elementos humanos implicados, docentes y educandos, establecen unas relaciones adecuadas, en las que interaccionen, y compartan objetivos y responsabilidades.

A nivel de la vida profesional del docente $\mathrm{y}$, más concretamente, en sus necesidades y expectativas, se pueden indicar una serie de frustraciones relacionadas con las demandas de la sociedad actual hacia el sistema educativo y las posibilidades reales de respuesta. 
Una de las contradicciones se encuentra en que, si bien es cierto los nuevos impulsos de la psicopedagogía sitúan al estudiante como el verdadero protagonista de su propio aprendizaje, los docentes no han acertado del todo a despegar su imagen del modelo tradicional, en el cual el profesor o la profesora son responsables de todo el proceso -tanto de la planificación, como del hecho de que cada joven lleve a su mejor término el proceso de enseñanza-. Así, pues, de una manera casi sistemática, se establecen relaciones de tipo jerárquico con los educandos. Estas se adaptan muy mal a las nuevas tendencias y necesidades de orientación, motivación y contextualización, requeridas en la actualidad.

Para satisfacer estas necesidades, sería preciso adoptar unas posibilidades de trabajo distintas y desarrollar una mayor capacidad para establecer, con los educandos, una auténtica comunicación en profundidad. Tales requerimientos no siempre son manejados por los docentes, ya que en muchos casos carecen de vocación e inteligencia interpersonal e intrapersonal. De esta manera, se encuentra una de los mayores fuentes de problemas y de contradicciones para una profesión en la que se tienen que conjugar elementos tan dispares como las relaciones de sentimientos, afectos y el contacto con la distancia que imponen la justicia e igualdad en el trato (Gil-Monte y Peiró, 2000). También cabe mencionar la exigencia de responder a las regulaciones y a la documentación administrativa, las cuales, en muchas ocasiones, no tienen nada que ver con las realidades inmediatas que configuran la labor cotidiana del docente y la docente.

A pesar de todas estas realidades en que se ve inmerso el docente, este continúa estando solo frente a su clase. En contraste, cada día se le solicita formar equipo con sus colegas y asumir tareas que exigen la cooperación con muchos especialistas: equipos de orientación, planificación, tareas inherentes (actividades para recolectar fondos), trato con los padres y madres, con otros docentes, con la administración, etc. Junto a todo esto, los profesores y las profesoras perciben que la imagen social de su profesión está cada día más deteriorada y es objeto de múltiples críticas. Socialmente parece haber una concepción errónea, en cuanto a que si los estudiantes no aprenden, es porque el profesor no enseña bien. Las expectativas que se derivan de este supuesto son fuente de culpabilidad, frustración y fracaso.

Son muchos los sectores que tienen ingerencia sobre esta labor: los políticos, los que se concentran en el área social, el sector económico, sectores comunales, las propias familias, mismos que en un momento u otro se manifiestan muy críticos con los docentes en general y cuestionan la eficacia del profesor y profesora, sin realizar un verdadero ni profundo análisis de los factores determinantes de tan compleja tarea. De forma cada vez más generalizada, y a causa de demandas excesivas, contradictorias y ambiguas, no se consigue el equilibrio deseable entre lo que la sociedad pide y espera, y lo que el profesional de la docencia puede ofrecer según sus capacidades y competencias. Tal situación, que se da con frecuencia en el mundo educativo, puede llegar a desencadenar la insatisfacción docente y cuadros de ansiedad que dificultan o impiden un correcto ejercicio de la profesión y que afectan negativamente a los educandos y, por ende, la calidad de la docencia.

Por el contrario, si este equilibrio se produjera, el nivel de satisfacción sería alto y la autoimagen profesional positiva. A pesar de lo indicado, existen muchos profesionales satisfechos de su labor, quienes asumen las inevitables e innumerables dificultades del trabajo cotidiano, aciertan al desempeñar su rol profesional y establecen unas interacciones personales positivas y productivas con los educandos, los colegas y su entorno más inmediato. En tal caso, tanto en los profesores y profesoras que pueden subsistir sin "quemarse", como en los potencialmente "quemados", se evidencia la importancia de ejercer su profesión desde un enfoque interdisciplinario, lo más amplio posible.

Se puede destacar que todas las circunstancias anteriormente descritas propician que el profesorado se sienta, personalmente y profesionalmente, abrumado y desconcertado, con 
fuertes contradicciones entre sus derechos y deberes. Con todo ello, la enseñanza como profesión tiene algunos rasgos característicos que contribuyen a intensificar la problemática del Burnout (Manassero, Fornés, Fernández, Vazquez y Ferrer, 1995) y que son los siguientes:

- Exigencia de un contacto e interacción personal constantes con los estudiantes, que debería caracterizarse por ser paciente, sensible y útil.

- El trabajo docente esta siempre abierto al examen y evaluación por parte de diferentes sectores e intereses.

- La actividad docente se ejerce con personas (los educandos) con unas motivaciones e intereses propios.

- La práctica docente ofrece pocas oportunidades a los profesores y profesoras para relajarse, descansar y entablar relaciones con otros adultos a lo largo del horario laboral.

- La remuneración salarial es siempre menor que la de otros puestos de trabajo equivalentes por titulación o formación.

\section{Incidencia en secundaria}

Desde el punto de vista de Fuentes et al. (1997), este síndrome es más frecuente en docentes de secundaria que en los maestros de primaria. Esta situación se puede atribuir a que los educandos que acuden a este nivel se encuentran en la etapa de la adolescencia y, por su misma naturaleza, demandan mayor atención y al mismo tiempo pueden presentar mayores problemas de conducta o conflictos con los profesores y las profesoras. Además, la cantidad de educandos en secundaria es mucho mayor, por lo cual se aumentan las posibilidades de contacto con ellos y con sus padres. O, al contrario, se aumenta el desconocimiento de la realidad de cada educando, con la consiguiente impotencia de atención individualizada, situación que puede repercutir en el descontento profesional.

Por otra parte, Sevilla y Villanueva (2002) reportan que la formación académica de los docentes de secundaria no coincide con las expectativas reales de su responsabilidad. Es decir, la labor del docente implica mucho más que poseer el conocimiento de un área en particular, sino que acorde a la realidad actual debe ser psicólogo, sociólogo, madre o padre, médico o enfermero, sexólogo, entre muchas otras necesidades presentes en el proceso de dar respuesta a esta gran cantidad de requerimientos. Todo ello contribuye a que el profesional de la educación media se pueda ver afectado por el síndrome.

Aunado a lo antes expuesto, se puede considerar que la educación secundaria debe brindar a los estudiantes una formación básica y coadyuvar en la formación de una personalidad integral que les permita continuar los estudios superiores o, para quienes ya no continúan su formación, integrarse a la vida laboral. Al respecto, la responsabilidad del docente es fundamental y el riesgo de presentar problemas derivados de esta podría ser mayor y reflejarse en la calidad de la enseñanza.

\section{Conducta profesional}

Siguiendo las observaciones realizadas por diversos autores (Esteve, 1995; Manassero et al., 1995; Mingote, 1998), la conducta profesional del docente afectado por el síndrome de Burnout presenta las siguientes manifestaciones específicas: 
- Se muestran desmotivados y han perdido la paciencia, el optimismo y el interés por sus educandos.

- Manifiestan actitudes autoritarias, intolerantes o rígidas. La pérdida de paciencia, anteriormente referida, puede conducir a estas actitudes y ser fuente de problemas de variada índole.

- Buscan y necesitan vías y excusas para desmarcarse de la implicación en el trabajo y suelen adoptar actitudes contestatarias respecto a sus superiores.

- Perciben a los estudiantes y a sus familias de una manera cínica, como si atentasen contra su propia supervivencia o sus necesidades.

Se ha sugerido la existencia de tres prototipos de profesores (Manassero et al., 1995) que, aunque no deben tomarse como modelos exactos de la realidad, pueden ayudar a la comprensión de las manifestaciones más directamente observables en la conducta del educador afectado por el síndrome de Burnout:

1. Docentes agotados. Son los que reaccionan ante las excesivas demandas y los fracasos en la enseñanza trabajando cada vez menos, puesto que no esperan alcanzar ningún objetivo. Están desmotivados y, consecuentemente, trabajar más y mejor no tiene para ellos ningún tipo de compensación. Los profesores y profesoras agotados han cortado o eliminado su compromiso y su dedicación personal con la enseñanza.

2. Docentes frenéticos. Suelen ser docentes muy comprometidos con su trabajo, que reaccionan ante la adversidad y los fracasos sin reconocerlos y sin darse tregua. Incrementan más y más sus esfuerzos ante la adversidad, en lugar de racionalizar y dosificar sus fuerzas o modificar sus objetivos. El Burnout suele aparecer en sus vidas bruscamente, repentinamente, ya sea ante un fracaso importante como en un momento de debilidad. Cuando su sentido de la eficacia desparece, la desilusión lo invade todo. El agotamiento total, que había sido obviado por la ilusión de unos éxitos ficticios, se hace dominante en sus vidas. Este prototipo está muy relacionado con el modelo sugerido por Freudenberger (1974), cuando caracteriza a docentes extra-comprometidos y celosos de su trabajo, quienes incluso olvidan sus propias necesidades, se esfuerzan intensa y frenéticamente hasta que se agotan. Lo cierto es que en este prototipo se distingue un subtipo de profesor y profesora caracterizado, además, por el rasgo de frenesí, por el narcisismo-autosuficiencia y la impaciencia con los demás. A este subtipo pertenecen aquellos docentes que se creen indispensables e insuperables. El perfil corresponde a un tipo de persona emprendedora que hace siempre las cosas porque está convencida que si no las hace él nadie las haría. Con frecuencia sus educandos tienen una opinión positiva de él. Se destaca como aspecto positivo su hiperactividad. En cambio, puede resultar nocivo para sus compañeros y colegas, porque los juzgan intolerantemente por su propio entusiasmo y consideran que están poco dedicados o comprometidos. La diferencia fundamental, entre los profesionales agotados y los frenéticos, es que estos últimos se sienten tan absolutamente seguros de sí mismos que no se percatan de sus fracasos parciales hasta que llega el momento del gran derrumbe. Los agotados, por el contrario, son más conscientes de sus fracasos parciales y más comprensivos con la acomodación de sus colegas.

3. Profesores poco incentivados. Son aquellos que no encuentran en su trabajo ninguna motivación. No se sienten agotados ni estresados ni tienen afectada su autoestima. Estos docentes no perciben en su trabajo nada que consideren una buena recompensa y entienden que sus capacidades están infrautilizadas. La enseñanza, tal y como ellos la vivencian, no les aporta estímulos que recompensen el esfuerzo que hacen y se cuestionan las posibilidades de cambiar de trabajo. El origen de los poco incentivados es típico del Burnout, consideran que reciben mucho menos de lo que dan a la profesión, lo que genera una pérdida total de interés. 
La anterior caracterización, puede cambiar en la realidad, por las características propias de cada individuo y por el estadio del síndrome.

\section{Algunos factores individuales que pueden influir en el proceso del síndrome}

\section{Aspectos de la personalidad}

Las características de la personalidad configuran un elemento que debe ser considerado en relación con la intensidad y frecuencia de los sentimientos. Por ejemplo, la existencia de sentimientos de altruismo e idealismo acentuados puede facilitar el proceso de Burnout. Según Maslach y Jackson (1981); Tonon (2003), el trabajador con predisposición a "quemarse" se caracteriza por una serie de rasgos tales como: ser entusiasta, con mayor dedicación profesional, susceptibles a identificarse con los problemas de los demás, sensibles, obsesivos y con baja autoestima; pero, a la vez, excesivamente idealistas.

Este idealismo y los sentimientos altruistas llevan a los profesionales a implicarse excesivamente en los problemas y convierten en un reto personal la solución de los mismos (Menéndez y Moreno, 2006). Todo ello va a conllevar que se sientan culpables de los fallos, tanto propios como ajenos, lo cual repercutirá en bajos sentimientos de realización personal en el trabajo.

La mayoría de las veces, los profesionales que eligen un trabajo de servicio humano, como la enseñanza, comparten una característica común: son personas que poseen un desarrollo de la inteligencia interpersonal, es decir, son personas especialmente sensibles a las necesidades de los demás. Es en este sentimiento de base altruista, pero en exceso idealista, donde se sitúan algunos de los orígenes del Burnout. Ciertamente los profesionales guiados por esta voluntad de servicio tienen un mayor nivel de empatía hacia los demás y sufren más con las diferentes problemáticas y, en consecuencia, experimentan un mayor desgaste emocional. Si el individuo en cuestión no puede afrontar eficazmente las situaciones estresantes que va vivenciando, esto le conducirá al fracaso profesional $y$, por ende, al fracaso de las relaciones interpersonales. Estas características son propias del patrón de personalidad tipo A, utilizado en la psicología de la personalidad para aludir a aquellos sujetos que se caracterizan por manifestar impaciencia, velocidad excesiva, urgencia temporal, esfuerzo por el logro, competitividad, habla y gestos bruscos, así como compromiso excesivo por el trabajo. El patrón tipo A es definido, en gran medida, como opuesto al denominado patrón B, y se asume que este está más exento de estrés. Lo cierto es que los sujetos pertenecientes al patrón tipo B también experimentan estrés; pero la diferencia entre ambos está en que, en el tipo B, la forma de satisfacer sus necesidades de éxito y logro personal y profesional no origina los estragos físicos y psicológicos que se dan en el tipo A (Menéndez y Moreno, 2006 citando a Friedman-Rosenman).

\section{Expectativas personales}

En los procesos del síndrome de Burnout, las expectativas personales sobre el trabajo ocupan un papel central (Manassero et al., 1994). Algunas de las más frecuentes, en el ámbito de la enseñanza, son las siguientes:

- La confianza absoluta e ilimitada en la capacidad profesional (idealismo narcisista).

- La creencia de que realizar servicios extraordinarios para los educandos conllevará beneficios extraordinarios para ellos y para el propio profesor o profesora. 
- La expectativa de que todos los estudiantes tendrán una respuesta positiva ante la intervención personal.

- La impaciencia por ver los resultados del trabajo inmediatamente o con un margen temporal demasiado breve.

- La confianza en que los educandos se comporten según cierta expectativa del docente y la docente y les permitan ejercer su autoridad.

- La necesidad de que su trabajo sea apreciado y valorado por sus compañeros (as) y superiores inmediatos y especialmente por el estudiantado y sus padres y madres.

- El deseo de ejercer la docencia en un ambiente de trabajo estimulante y recompensado, pero la realidad es muchas veces opuesta.

- Deseo de recibir preparación formal para la innovación y los cambios. La ausencia de esta preparación suele comportar otro elemento de desencanto.

De todo lo expuesto hasta el momento se deduce que el factor subjetivo es primordial. Las expectativas frustradas o que no corresponden con la realidad son, en gran medida, el desencadenante de que se pierda el entusiasmo por la profesión.

\section{La vivencia del estrés}

El término estrés es muy usado en la actualidad. Generalmente se asocia a efectos negativos; pero, en alguna medida, potencia consecuencias positivas, ya que puede ser un estimulante. Al respecto, resulta importante que cada individuo descubra su nivel óptimo de estrés y no lo sobrepase, pues tal como lo indican Menéndez y Moreno (2006):

El estrés se manifiesta, de modo general, como una respuesta de activación anormal de algunos sistemas del organismo (hipotálamo-hipofisio-suprarrenal, autónomo simpático, médula suprarrenal) con inhibición de la glándula tímica cuando el equilibrio del organismo (homeostasis) se ve alterado por algún agente interno y externo. (p. 134)

Es decir, es un mecanismo de defensa ante un determinado estímulo. Como ya se mencionó, no siempre es malo: se puede tornar negativo en determinadas circunstancias frecuentes en ciertos modos de vida y desencadenar problemas graves de salud. Cuando esta respuesta natural se da en exceso, se produce una sobrecarga de tensión. Esta repercute en el organismo y provoca la aparición de enfermedades y anomalías patológicas que impiden el normal desarrollo y funcionamiento del cuerpo humano. En este sentido, la mayoría de los profesionales indican trabajar bajo estrés, situación más potenciada en quienes ejercen la docencia, por las características propias de la labor, sus expectativas personales y sociales, por su personalidad, entre otras.

\section{Problemática del rol}

El docente y la docente deben proyectar, poner en ejercicio, medir y adecuar permanentemente las estrategias para el desarrollo integral de la persona (maduración personal, moral y cívica), por medio de la promoción del aprendizaje y la construcción de saberes, habilidades y actitudes de los educandos, más allá de la mera instrucción, transmisora de conocimientos normalizados, propia de 
un sistema de enseñanza tradicional, logo céntrico en el cual se ha formado la generación actual de docentes. Este rol requiere de profesionales comprometidos, social e institucionalmente, que sean capaces de diseñar líneas de intervención que surjan de interpretar realidades, definir problemas, actuar dentro de márgenes no definitivos y ante circunstancias específicas, únicas e irrepetibles. El docente y la docente ya no ejercen su labor en solitario, deben conformar equipos humanos y laborales que les permitan formarse, replantearse sus prácticas y reflexionar sobre ellas.

Junto a lo anterior, la sociedad exige al docente y a la docente que tengan sentido crítico con respeto a sí mismos, a los contenidos culturales y al contexto social; que sean objetivos, democráticos y con vocación de servicio; que tiendan al trabajo cooperativo y amen su institución y sus estudiantes; que se sientan parte viva de la comunidad en que interactúan; que se esfuercen por alcanzar una conducta racional y científica; que manifiesten preferencia por el orden y la sistematización y que, sobre todo, sean muy creativos para desempeñar su formación y su experiencia al servicio del estudiante y de la institución, ambos parte de su zona de desarrollo próximo.

La multiplicidad de funciones que debe desempeñar el docente y la docente, de acuerdo con Esteve (1995); Travers y Cooper (1997), pueden incluir la de formular diagnósticos, hacer de consejeros, desempeñar funciones terapéuticas, desempeñar funciones administrativas, ser evaluadores, hacer informes de los educandos, y finalmente la de docentes; todo ello puede llevar a los conflictos interrol, surgidos cuando los docentes y las docentes tienen que asumir distintas funciones dentro de la institución. Este panorama refleja una situación en la que se dedica menos tiempo a la función docente. Cada vez se les exige a los profesores y profesoras que dediquen más tiempo a tareas administrativas y menos a la función propia del docente. Vale rescatar que este problema de rol no se debe confundir con el mobbin ya que este implica "(...) una situación continuada de acoso moral volcado sobre un individuo de modo que siente la presión del entorno (...)” (Menéndez y Moreno, 2006, p. 155).

\section{Edad, años servidos, sexo y estado civil}

En cuanto las variables de edad y años de ejercicio profesional, no está bien definida la relación existente con el Burnout. Si bien Tonon (2003) indica que, a medida que los años avanzan, el sujeto va ganando en experiencia y seguridad en las tareas laborales y puede mostrar, en consecuencia, una menor vulnerabilidad; en contraste, otros autores sostienen que, en los primeros años de trabajo, el educador o educadora es más vulnerable, por las expectativas que lleva de poder realizar un trabajo integral, de cooperación y calidad. Es decir, sus propias exigencias son mayores. Por otro lado, Borg y Falzon (1989); Van Ginkel (1987) proponen que los docentes y las docentes más experimentados (as), con más de veinte años en la profesión, pueden tener una respuesta de estrés mayor que la de sus compañeros (as). En relación con la variable sexo, autores como Peiró, Luque y Meliá (1991); Travers y Cooper (1997) coinciden en que las mujeres están expuestas a mayores situaciones de tensión, puesto que, en su mayoría, realizan una doble jornada: la laboral y la familiar. En cuanto al estado civil, este no es determinante, pues no es el estar casado o soltero lo que influye, sino el nivel de apoyo socio emocional que brinde la familia o personas con las que convive (Tonon, 2003).

\section{Factores de riesgo en el contexto laboral}

Como ya se ha indicado, pueden ser muchos los factores que inciden en este síndrome. A continuación se describen algunos, según Maslach (1982): 


\section{La baja implicación laboral establecida por un determinado modelo organizacional}

La satisfacción laboral, según Peiró et al. (1991), es uno de los indicadores más clásicos y al que con mayor frecuencia se recurre cuando se persigue conocer cuál es la actitud general de las personas hacia su vida laboral. De algún modo, las personas, y en este caso los profesionales y las profesionales de la educación, tienen una idea más o menos elaborada de cuál es el tipo de trabajo que les gusta, cómo debería llevarse a cabo, en qué tipo y estilo de institución y con qué condiciones. De esta manera, las personas pueden comparar su idea de cómo deberían ser las cosas con su realidad presente y cotidiana. De tal comparación surge un juicio y una actitud asociada hacia su trabajo y hacia los demás aspectos importantes de su vida laboral y, en consecuencia, se establece un determinado grado de satisfacción o insatisfacción. Las consecuencias de tal satisfacción o insatisfacción pueden afectar la cantidad y la calidad del trabajo que desempeñan.

Las actitudes del alumnado y su comportamiento constituyen causas de estrés entre el profesorado. Las exigencias a las que se enfrentan los docentes y las docentes han experimentado un cambio de gran magnitud en los últimos años, lo cual se manifiesta en un aumento importante de las responsabilidades que recaen sobre ellos, sin que paralelamente la familia y la comunidad en general lo hayan asumido, o les hayan ayudado. Lo cierto es que cada vez más se aprecia la tendencia de delegar la responsabilidad educativa en los profesores y las profesoras, en detrimento del papel de la familia, como eje de la sociedad y primera fuente de enseñanza. También se han producido cambios, a menudo en perjuicio del profesorado, respecto a las actitudes de la sociedad hacia la disciplina educativa.

\section{Sobrecarga laboral}

Otro aspecto de la profesión docente que se considera relacionado con el exceso de trabajo y, consecuentemente, con la aparición del estrés es el problema de tener, dentro de una misma clase, educandos con una amplia gama de capacidades diversas y necesidades educativas diferenciadas. Esto suele requerir una mayor planificación de las clases y una evaluación más detallada, así como atención personalizada. Además se enfrentan otras tareas inherentes al cargo: reuniones, efemérides, documentación de los estudiantes, actividades para recolectar fondos, entre otros. Esa sobrecarga laboral se relaciona con las presiones derivadas del tiempo, no solo en términos de la cantidad de trabajo que deben realizar cada día, sino también en cuanto a la cantidad de tareas que deben llevarse a sus casas, lo cual interfiere en sus vidas privadas.

\section{Burocratización}

Otro aspecto a considerar, según Fernández, (1994); Gil-Monte y Peiró, (1997), es que los docentes realizan su trabajo en organizaciones que responden a un esquema de burocracia profesionalizada. Para ser verdaderamente funcionales, tales burocracias profesionalizadas requieren una serie de condiciones en el ambiente y en el clima organizacional. Respecto al poder y la jerarquización, estas organizaciones demandan una distribución democrática del poder, de forma que el propio profesional ejerza el control sobre su trabajo y las decisiones que le afectan. En tal sentido, se deben evitar las desigualdades en el trato por parte de los administradores (as), y dejar de lado la libertad de acción con unos sí y con otros no. 


\section{Ambiente laboral}

El edificio, las instalaciones y el mobiliario conforman el ámbito dentro del cual se establecen las relaciones en las que se desarrolla la tarea educativa. Como espacio material, el edificio y sus instalaciones conforman un conjunto de condiciones que pueden afectar la calidad de la enseñanza y del aprendizaje. Por una parte, generan sensaciones de comodidad o incomodidad, seguridad o peligro, potencialidad o carencia y, con ellas, comprometen la posibilidad o dificultad de los sujetos para sentirse, en mayor o menor grado, "predispuestos" frente a las demandas del trabajo. De la misma manera, del modo en que facilitan o bloquean el movimiento y el intercambio, inciden también en la riqueza y diversificación de los comportamientos, tanto de los educandos como de docentes.

Por otra parte, la calidad y diversificación de las instalaciones y del material didáctico afectan directamente al tipo y complejidad de las experiencias curriculares en las que participan docentes y educandos, ya que es bien sabida la complicación que significa un espacio inadecuado, con poca luz, con mucho ruido, mal equipado y el sobreesfuerzo que exige trabajar como si esa limitación no existiera.

\section{Consecuencias}

Como se ha venido indicando, los profesores y las profesoras, en general, tienden a implicarse tanto en su trabajo y se sienten tan moralmente comprometidos en la enseñanza, que cuando se les presiona o perciben que tienen que actuar de manera contraria a sus valores y sentido de la identidad profesional, se muestran estresados, con sentimientos de culpa y pérdida de autoestima. En consecuencia, tienden a ignorar sus propias necesidades, a cuidarse poco, pues consideran que las posibles satisfacciones de la enseñanza dependen de atender y sacrificarse más por las necesidades de otros que por las de ellos mismos.

\section{Consecuencias sobre el individuo}

Consideradas desde la perspectiva individual (Gil-Monte y Peiró, 1997), las consecuencias del estrés, asociadas al síndrome de Burnout en docentes, producen un deterioro en la salud de los profesionales y en sus relaciones interpersonales, tanto dentro como fuera del ámbito laboral. A su vez, también producen una repercusión negativa en la calidad de la docencia. Las principales manifestaciones se centran en la presencia de sentimientos de desgaste, tensión, irascibilidad, nerviosismo, fatiga extrema. Las principales consecuencias del malestar docente sobre la personalidad de los profesores y las profesoras, centradas en su dimensión individual, de acuerdo con Esteve (1995), recorren una amplia escala, que pueden variar tanto en su manifestación como en su intensidad, y se agrupan en:

1. Sentimientos de desconcierto e insatisfacción ante los problemas reales de la práctica cotidiana de la enseñanza.

2. Peticiones de traslado o cambio como forma de huir de situaciones conflictivas.

3. Desarrollo de esquemas de inhibición como forma de cortar la implicación personal con el trabajo que se realiza. 
4. Deseo, implícito o manifiesto de abandonar la docencia.

5. Ausentismo laboral, como mecanismo para cortar la tensión acumulada.

6. Agotamiento como consecuencia de la tensión acumulada.

7. Estrés.

8. Ansiedad.

9. Depresiones.

10. Autoculpabilización ante el rendimiento de los educandos.

11. Ansiedad como estado permanente.

Así pues, ante las presiones de las diversas fuentes de tensión presentes en la enseñanza, potenciadas por la aceleración del cambio social, los profesores y las profesoras ponen en juego diversos mecanismos de defensa, como son los esquemas de inhibición y rutina, o el ausentismo laboral, que presentan el aspecto negativo de bajar la calidad de la educación, pero que sirven para aliviar, momentáneamente, la tensión del profesional.

\section{Consecuencias sobre la organización}

Tales conductas disruptivas tienen repercusiones no solo en el trabajador, sino también en la institución educativa, tal y como se indicó antes. Lo cierto es que cuando los trabajadores de un centro escolar comienzan a padecer los síntomas del síndrome de Burnout, las consecuencias para la institución pueden ser realmente importantes. Las conductas y las actitudes laborales alteradas que presentan los sujetos quemados, así como el deterioro en la salud física y psíquica, van a incidir directamente en el funcionamiento y rendimiento de la institución y esto se reflejará en el empeoramiento de la atención del estudiantado. Junto a todo lo citado, el disconfort subjetivo que siente la persona afectada conlleva situaciones de conflicto con el profesorado, demandas de traslado y, ocasionalmente, cese o separaciones de la práctica profesional. Las graves repercusiones individuales que produce el Burnout afectan las organizaciones educativas, sobre todo influyen en la deshumanización y en la calidad de la enseñanza.

\section{Medidas para prevenir el síndrome de Burnout}

\section{En el nivel personal}

No se puede plantear el manejo del Burnout sin abordar el propio esquema de ver las cosas, sin una modificación conveniente de actitudes y aptitudes. Se debe tener un proceso adaptativo entre las expectativas iniciales con la realidad que se impone, marcando objetivos más realistas, que permitan, a pesar de todo, mantener una ilusión por mejorar sin caer en el escepticismo.

Se impone un doloroso proceso madurativo en el que se acepten los errores y limitaciones, con frecuencia a costa de secuelas y cicatrices en el alma. Se debe aprender a equilibrar los objetivos de esta labor (adecuación eficiente de los escasos recursos disponibles, pero sin renunciar a lo más valioso de esta profesión: los valores humanos).

En cualquier profesión y más en docencia es trascendental equilibrar las áreas vitales: familia, amigos, aficiones, descanso, trabajo y evitar, a toda costa, que la profesión las absorba, pues los vínculos sociales: familia, amigos, aficiones y descanso, son grandes protectores del Burnout. 


\section{En el nivel institucional}

\section{Equipos de trabajo}

Los compañeros de trabajo tienen un papel vital en el Burnout:

- En el diagnóstico precoz: son los primeros en darse cuenta antes que el propio interesado.

- Como una importante fuente de apoyo: son quien mejor los comprenden, ya que pasan por lo mismo. Por el contrario, cuando las relaciones son malas, contribuyen a una rápida evolución del Burnout.

Por todo esto es de vital importancia fomentar una buena atmósfera de trabajo:

- Facilitando espacios comunes no informales dentro de la jornada laboral.

- Promoviendo la colaboración y no la competitividad (objetivos comunes).

- Formando grupos de reflexión sobre temas emocionales, tanto de las relaciones docentedocente, docente padres de familia como docente-educando.

\section{Administrativo}

Otro ente que puede hacer su aporte en la prevención es la parte administrativa de la institución, la cual puede:

- Limitar una agenda máxima de trabajo y tener un ritmo cotidiano de trabajo eficiente.

- Minimizar y facilitar la burocracia.

- Apoyar con asesoramientos específicos, para mejorar el proceso de enseñanza y aprendizaje.

- Coordinar actividades académicas en conjunto.

- Disminuir la cantidad de estudiantes por grupo, para poder conocerlos mejor y, por ende, mejorar la comunicación con ellos y con sus padres- madres.

- Desarrollar programas del manejo y control de estrés con especialistas.

- Liberar al profesional, cada 5-7 años, unos meses de la docencia, para formación continua y para que reciba capacitación acorde con sus necesidades o, simplemente, para descansar de su rutina. Así se aumenta su competencia, y, a su vez, se influye en su motivación.

- Coordinar con las instancias pertinentes para que se guíe al docente y a la docente en el trato con adolescentes.

- Implementar a una imagen positiva del profesor y profesora.

- Establecer un balance entre las políticas educativas y la realidad, así como con la autonomía de los centros educativos.

\section{Algunas ideas para concluir}

- El docente y la docente de secundaria deben adecuar constantemente las estrategias para el desarrollo integral del grupo de adolescentes, por medio de la promoción del aprendizaje y de la construcción de saberes, habilidades y actitudes, más allá de la mera transmisión de conocimientos normalizados. 
Esta necesidad requiere profesionales comprometidos, social e institucionalmente, que sean capaces de diseñar líneas de intervención, previo análisis contextual. Este profesional ya no ejerce su labor en solitario, debe conformar equipos humanos y laborales que le permitan formarse, replantearse sus prácticas y reflexionar sobre ellas, es decir, debe ser capaz de formar competencias personales y profesionales para evitar quemarse, pues la sociedad cada día demanda más.

- Se puede destacar que junto con los elementos de tipo individual, algunas de las causas de Burnout están en la desvalorización del trabajo del docente y la docente de secundaria, evidente en nuestro contexto social, lo cual converge en profesionales de Educación Media insatisfechos con su propia labor o en personas que hacen un gran esfuerzo por tratar de cumplir con todos los elementos curriculares y extracurriculares.

- En los momentos de desarrollo tecnológico y cambio social en que nos encontramos, y ante demandas excesivas, contradictorias y ambiguas, no se consigue el equilibrio deseable entre lo que la sociedad pide y espera, y lo que el profesional de la docencia puede ofrecer, acorde a sus capacidades y competencias, por lo cual se debe tener presente que lo fundamental en la vida es ser feliz con lo que se hace y disfrutar cada momento. Toda labor se debe asumir con respeto, seriedad, honestidad, y sobre todo la que implica el trato con seres humanos, pues en las manos de los docentes y las docentes se encuentra la sociedad.

- El estrés es una respuesta natural y necesaria para la supervivencia, lo que no es normal es adquirirlo como un estilo de vida, pues esto acarrea problemas de salud. Razón por la cual, como es parte de la vida y del ejercicio profesional, lo que se puede hacer es nivelarlo, por ejemplo, en lo personal, estableciendo momentos de ocio, permitiendo compartir con amigos, familiares, seres queridos; a nivel de instituciones formadoras, dando mayor importancia a la parte psicosocial en el manejo de adolescentes, estableciendo metas reales, sin crear en el futuro docente falsas expectativas de su labor; en cuanto a la administración, tratando de manejar las relaciones humanas de manera profesional, velando porque se elija con vocación un trabajo trascendental y reconfortante como es la docencia.

\section{Referencias bibliográficas}

Borg, M. G. y Falzon, J. M. (1989). Stress and Job Satisfaction Among Primary School Teachers in Malta [El estrés y la satisfacción profesional entre los maestros de escuela primaria en Malta]. Educational Review, 41(3), 271-279.

Esteve, J. M. (1995). Los profesores ante el cambio social. Repercusiones sobre la evolución de la salud de los profesores. Barcelona: Anthropos.

Fernández, L. (1994). Instituciones educativas. Dinámicas institucionales en situaciones críticas. Barcelona: Paidós.

Fuentes, J. M., Quevedo, M. P, Velasco, C., Sánchez, A., Sánchez, T., Salgado, A. .. Yela, J. R. (1997). El síndrome del "Burnout": estudio empírico en profesores de enseñanza primaria. Iberpsicología: revista electrónica de la Federación española de Asociaciones de Psicología, 2(1). 
Gil-Monte, P. y Peiro, J. M. (1997). Desgaste psíquico en el trabajo. El síndrome de quemarse. Madrid: Síntesis.

Gil-Monte, P. y Peiro, J. M. (2000). Un estudio comparativo sobre criterios normativos y diferenciales para el diagnóstico del síndrome de quemarse por el trabajo (Burnout según el MBI-HSS en España. Revista de Psicología del trabajo y de las organizaciones, 16(2), 135-149.

Manassero, M. A., Fornés, J., Fernandez, M. C., Vázquez, Á. y Ferrer, V. (1995, setiembrediciembre). Burnout en la enseñanza: análisis de incidencia y factores determinantes. Revista de Educación, 308, 241-267.

Manassero, M. A., Vázquez, A., Ferrer, V., Fornés, J., y Fernández, M. C. (1994). Burnout en profesores: impacto sobre la calidad de la educación, la salud y consecuencias para el desarrollo profesional (Memoria final de investigación). Madrid: MEC-CIDE.

Maslach, C. (1982). Burnout. The Cost of Caring [Burnout. El costo de la atención]. Englewood Cliffs, NJ.: Prentice Hall.

Maslach, C. y Jackson, S. (1981). The measurement of experienced burnout [La medición del desgaste sufrido]. Journal of Occupational Behavior, 2(99), 99-113.

Menéndez, C. y Moreno, F. X. (2006). Ergonomía para docentes. Análisis del ambiente de trabajo y prevención de riesgos.Graó: España.

Mingote, J. C. (1998). Síndrome de Burnout o síndrome de desgaste profesional. FCM. 8, 493-503.

Peiró, J. M., Luque, O. y Meliá, J. L. (1991). El estrés de enseñar. Sevilla: Alfar.

Sevilla,U. y Villanueva, R. (2002). La salud laboral docente en la enseñanza pública. Gabinetes de estudios y de salud laboral [2 Acción Sindical] España: Publicaciones de la de la Federación de enseñanza de comisiones obreras Recuperado de http://www.uv.es/ccoo/downloads/lsalutlaboraldocenteenlaenspublica.pdf

Tonon, G. (2003). Calidad de vida y desgaste profesional: una mirada del síndrome de Burnout. Buenos Aires: Espacio.

Travers, A. y Cooper, C. (1997). El estrés de los profesores. La presión en la actividad docente. Barcelona: Paidós.

Van Ginkel, A. (1987). Demotivatie bij leraren [Motivación de los profesores]. Lisse: Swets y Zeitlinger. 